\title{
Group decisions and the amount of transmitted information in absolute identification of pitch
}

\author{
ANTE FULGOSI, ZVONIMIR KNEZOVIĆ, and PREDRAG ZAREVSKI \\ Filozofski Fakultet, Zagreb, Yugoslavia
}

\begin{abstract}
Sixteen tones differing in frequencies from 80 to $4400 \mathrm{~Hz}$ were presented in random order 100 times each to five subjects for absolute identification. The amount of transmitted information (in bits) and the corresponding number of identified categories of pitch were determined on the basis of: (1) individual identification responses, represented by the median value of individual transmission indices; (2) the dominant responses given to each presented stimulus; and (3) the responses given by the group as a whole based on group agreement and group decision. The amount of transmitted information was larger in the case of the dominant responses than the median of the individual identification performance and was largest in the case of group decision (the percentage increases in the number of identified categories of pitch were $21.0 \%$, $37.0 \%$, and $14.7 \%$, respectively).
\end{abstract}

In a recent investigation (Fulgosi, Knezović, \& Zarevski, 1983), it was determined that the amount of transmitted information in absolute identification of pure tones differing in pitch is greater when it is calculated from the dominant (modal) responses of the group of subjects to each presented tone than when the median of the individual indices of transmission is taken as an indicator of the identification performance. The average percentage increase in the number of identified categories for the three experimental groups in Fulgosi et al. (1983) was $38.7 \%$. The results were explained as an indication that random factors influences individual identification performance, lowering the preciseness of the identification. Such factors are less effective when the identification is based on the responses of several subjects, and their joint identification performance is for this reason superior to that of the individual.

In the present experiment, we were interested in another kind of group identification procedure and another possible index of the group identification performance. The particular index and procedure that were investigated in the present experiment are based on the agreement of the members of the identification group and a collective decision about the identification response that should be given to the presented stimulus.

Previously investigated dominant or modal response (Fulgosi et al., 1983) could be treated as an "indirect" group identification response or an "indirect" index of the identification performance of the group, since it is determined on the basis of individual (direct) responses by each subject. To obtain a direct group response to each stimulus, we asked subjects to reach an agreement about which identification response they would give as a group after they had given their individual responses to the just presented stimulus.

The authors' mailing address is: Odsjek za Psihologiju, Filozofski Fakultet, 41000 Zagreb, Yugoslavia.
The interest was the same as in the earlier experiments. We suggest that individual identification performance is under the influence of random factors. Group performance (measured or expressed by the dominant or group response) should eliminate at least a part of these random influences, and, for this reason, group identification performance should be higher than individual identification performance.

In the present experiment we compared three indices of transmitted information: (1) amount of transmitted information calculated from individual responses, represented by the median of individual transmission values; (2) amount of transmitted information calculated from the dominant (modal) responses to each presented stimulus; and (3) amount of transmitted information calculated from responses given by the group as a whole and based on the group agreement.

\section{METHOD}

Subjects

Five subjects of normal hearing ability were included in the experimental group on a random basis. All subjects were students of psychology.

\section{Apparatus}

Tones of different frequencies were generated by an RC generator (Type MA 3604) that was connected to five Koss earphones.

\section{Stimuli}

Licklider's (1950) graph of the loudness of tones of different frequencies was used to select tones of approximately the same loudness. The following 16 tones were presented: 80, 120, $160,220,300,400,500,700,1000,1500,1800,2400,3000$, 3400,4000 , and $4400 \mathrm{~Hz}$.

\section{Procedure}

The experiment was conducted in a sound-attenuating room. Stimuli were presented in random order. Each stimulus was presented for $4 \mathrm{sec}$. The experiment was divided into $20 \mathrm{seg}$ - 
ments. In each segment, each tone was presented five times.

In the first 10 segments (the first half of the experiment), after each group identification response, the subjects were given feedback information (the right identification response). In the second 10 segments (the second half of the experiment), no feedback was given.

The subjects were asked to identify each tone with the proper number (from 1 to 16). Number 1 was used for the identification of the lowest tone $(80 \mathrm{~Hz})$.

The response-giving procedure was as follows. After each subject had written his or her own (individual) identification response in his or her protocol and after all subjects had entered their individual identification responses in their protocols, each member of the group informed all other members about his or her identification response. Next, all members discussed their differences and reached an agreement and decision about the response that should be given to the experimenter as their joint response.

The order of the announcement of the individual identification responses to other members of the group was circular. The subject who was first to announce his or her particular identification response to the other members was also the subject who later informed the experimenter about the group decision. The subjects were asked to reach agreement as soon as possible, but no time restrictions were imposed.

\section{RESULTS}

On the basis of individual and group responses, the following indices of transmitted information for the second half of the experiment were determined: (1) median value of individual amounts of transmitted information (M); (2) amount of transmitted information calculated from the dominant (modal) responses (D); and (3) amount of transmitted information based on the group decision $(\mathrm{G})$.

Table 1 shows amounts of transmitted information (in bits and corresponding categories) based on individual identification responses.

As can be seen in Table 1, the median value of the individual transmission performance is 2.64 bits, which corresponds to 6.2 different categories of pitch. The amounts of transmitted information calculated on the basis of the dominant (modal) responses and of the group responses (in bits and categories) are shown in Table 2.

Table 3 shows the increase in the amount of transmitted information expressed in percent between different types of identification responses.

Table 1

Amounts of Transmitted Information (in Bits and Categories) in Individual Identification of Pitch

\begin{tabular}{ccc}
\hline & \multicolumn{2}{c}{ Transmission } \\
\cline { 2 - 3 } Subjects & Bits & Categories \\
\hline 1 & 2.44 & 5.4 \\
2 & $2.64^{*}$ & $6.2^{*}$ \\
3 & 2.40 & 5.3 \\
4 & 2.66 & 6.3 \\
5 & 2.69 & 6.4 \\
\hline
\end{tabular}

*The median value.
Table 2

Amounts of Transmitted Information (in Bits and Categories) Based on Dominent and Group Decision Responses

\begin{tabular}{ccc}
\hline & \multicolumn{2}{c}{ Transmission } \\
\cline { 2 - 3 } Type of the Response & Bits & Categories \\
\hline Dominant & 2.90 & 7.5 \\
Group Decision & 3.10 & 8.6 \\
\hline
\end{tabular}

Table 3

Percent Increase of the Number of Identified Categories of Pitch in Different Types of Identification Responses

\begin{tabular}{ccc}
\hline \multicolumn{3}{c}{ Types of Responses Compared } \\
\cline { 2 - 3 }$(\mathrm{D}-\mathrm{M}) / \mathrm{M}$ & $(\mathrm{G}-\mathrm{M}) / \mathrm{M}$ & $(\mathrm{G}-\mathrm{D}) / \mathrm{D}$ \\
\hline 21.0 & 37.0 & 14.7 \\
\hline
\end{tabular}

Note-M = median of individual transmission indices; $D=$ transmission based on the dominant responses; $G=$ transmission based on group decision.

\section{DISCUSSION}

Both indices of the identification performance of the entire group (based on the dominant response to each stimulus and based on the group decision) are superior to the individual index (the median of the individual transmission indices). This means that the group identification of pitch of the pure tones is superior to the individual one.

Transmission of information by the dominant and group decision responses is even larger than the largest individual index of identification performance in the group (2.69 bits or 6.4 categories).

The increase in the amount of information transmitted by the dominant responses in comparison with that transmitted by the individual response (represented by the median of individual transmission values) in terms of categories is $21.0 \%$. This increase is smaller than the increase that was found in our earlier experiments (38.7\%) (Fulgosi et al., 1983). The difference possibly can be explained by the fact that the individual identification performance of the present group was higher than the identification performance of two of the three groups in the earlier experiments and was closer to the performance of the best group in our earlier experiments, for which we found an increase of only $27.4 \%$ (Fulgosi et al., 1983).

The main finding of the present investigation is a substantial increase in the amount of transmitted information in the responses based on group agreement and decision. This increase, in percentage of identified categories, is $37 \%$ in comparison with the median value of individual transmission indices and $14.7 \%$ in comparison with the dominant responses. It seems that group discussion, group agreement, and group decision are beneficial to the identification of pitch in absolute-judgment situations.

\section{REFERENCES}

Fulgosi, A., Knezović, Z., \& Zarevski, P. (1983). Amount of information transmitted in absolute judgments of pitch calculated according to the majority rule. Bulletin of the Psychonomic Society, 21, 193-194.

LickLider, J. C. R. (1950). Basic correlates of the auditory stimulus. In S. S. Stevens (Ed.), Handbook of experimental psychology. New York: Wiley.

(Manuscript received for publication December 13, 1983.) 